\title{
User Experience in Arabia: The Business Case for User-Centred Design and Usability Engineering
}

\author{
Ali al-Azzawi \\ Director of User Experience, HeiTech International (Dubai, UAE) \\ Visiting Research Fellow, Digital World Research Centre, University of Surrey (UK) \\ ali@ux.ae
}

\begin{abstract}
A common problem faced by technology projects is that while every effort is made to deliver at the highest quality, and ensuring well constructed business specifications, the outcome falls short of user expectations, rendering a system difficult to use, thereby negatively impacting adoption by the user. This paper highlights the need to involve the user at an early stage in the development process, using a User-Centred Design (UCD) approach and User Experience (UX) methods, and building low cost prototypes that encourage low cost optimisation, in order to mitigate the risk of later re-work, and increase the chances of holistic designs rather than incremental changes. The paper also highlights the view that a User Interface (UI) is in fact a business interface, and optimising the $\mathrm{UI}$ is synonymous with optimising business KPIs.
\end{abstract}

Keywords - User Experience, UX, Usability, User-Centred Design, UCD

\section{INTRODUCTION}

The concept of a 'User-Centred Business' is not new, though usually referred to as a 'customer focused business', where the business succeeds if it is oriented towards what the customer (user) wants. In this way, there is a high chance of the user adopting the product or service. This is a macro view, and is of course important and businesses usually spend significant effort conducting market research to establish the extent that their product will be adopted. What is not so normal, in Arabia, is to adopt a micro view, and explore the way the customer uses the product or service itself. This is odd considering that the product is the interface with the business, and if this interaction is not optimised, there will be a bottleneck impeding the success of the entire business.

The above issues relate to many reasons, some of which are historic norms in terms of how technological projects are implemented. The usual methods adopted involve Engineering being given a central role, and rightly so. After all, for a project to succeed, the technology has to work in an effective and efficacious way. The business owners also play a leading role. This is because if the project does not deliver the business goals, then it is deemed a failure (business is used here in a generic sense to include organisations, not just commercial). Nonetheless, to a very large extent, this is a valid and successful combination. The team then follows a process towards implementing projects. For example, IT development projects usually start with the business goals converted to a Software Requirements Specification (SRS) or similar, where a clear definition of what the business outcomes and processes are described, sometimes including use cases, within a common structure such as the Software Development Life Cycle (SDLC). Once there is agreement between all parties, these specifications are turned into operational details in the form of the Functional Specifications Document (FSD). The FSD then becomes the blueprint that the engineers and developers use to actually implement the work.

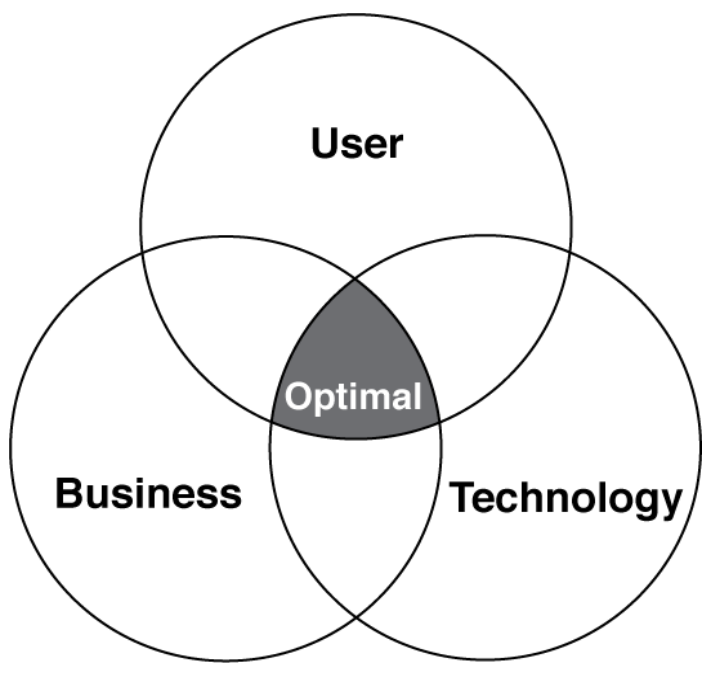

Figure 1: The three vital aspects of an optimised and successful IT project, that underpin a holistic and UserCentred Design approach. 
This basic approach has worked well in terms of delivering working systems that really do deliver the business goals - as they were articulated. However, all too often, stakeholders are surprised by a system that ticks all the boxes, yet has the users complaining. This is particularly frustrating for business owners, who made every effort to adhere to the highest standards for all aspects of the project. So, what is going wrong?

\section{BUSINESS, TECHNOLOGY \& USER}

The problem starts when business requirements are converted to the User Interface (UI). This conversion process relies on little theoretical, practical foundations, rationale, or sound methodology, that pays close attention the 'User' part of UI. Though there are exceptions where UX personnel are sometimes involved in the development of $\mathrm{UI}$ elements of the FSD, UX is usually absent. The process is usually done by one-to-one mapping of business and user requirements to UI features or elements. In extreme cases, for example, when a matrix of data is to be shown to the user, the developers will simply render a table on the UI. This is, in principle adequate as far as fulfilling the articulated business goals. However, there are many problems with this approach. For a start this promotes an iterative building process, with shifting specifications, which is expensive on development costs, where processes and interface designs are only rendered and tested after the initial development phases, in an almost trial and error fashion. This means that any practical problems with usage will be discovered after the expense of development, requiring subsequent re-work, which also slows down project progress. Also, another major issue is that later changes and re-work effectively become incremental changes, rather than being part of a design with any holistic view of the project. These incremental builds and changes eventually lead to a sub-optimal, fractional and siloed design, incurring more resource costs in development than necessary, and later increased support and maintenance costs.

Converting business goals to $\mathrm{UI}$ specifications is prone to suffer from the above mentioned problems. However, converting user needs to $\mathrm{UI}$ specifications (using UX methods), on the other hand, has distinct advantages. For a start, converting user goals and needs to UI has a sound psychology theory and practice with a substantial body of knowledge within the user experience, user research (Sharp, Rogers, \& Preece, 2007), usability and design industries (Kumar, 2013), including well-proven user research methodologies that take cultural variations into account (Al-Azzawi, 2010). Also, being focused on the user from the start, ensures that the system is one that the user will want, use and adopt.

The benefits of a user-centred approach rely on the simple equation of cost / benefit which users are constantly evaluating. The outcome of their evaluation would influence the rate of adoption and use of device or service (Davis, 1989; Davis, Bagozzi, \& Warshaw, 1989; Lund, 2011). However, 'cost' is not merely the financial cost, though this is important, it includes the cognitive load and effort required to use the technological artifact, i.e. the level of difficulty in use. Also, the 'benefit' is not entirely the actual real or direct benefit, but more importantly, perceived and implied benefits and value (Al-Azzawi, 2013). Therefore, a well designed system that increases the rate of adoption, is one that correctly uses sound psychology knowledgebase in the presentation of the system in the user interface, as well as the presentation of benefits in the way the 'user story' unfolds, including how the brand fits within the story.

Therefore, a more efficient and optimal approach is to create product specifications that combine user and business goals, and converting the result of this combination into a UI that addresses the needs of all stakeholders, including technological constraints (Figure 1). The User-Centred Design approach is well-established (Dix, Finlay, Abowd, \& Beale, 2004; Norman, 1990; Sharp et al., 2007), and starts with requirement gathering, including stakeholder interviews (business, user, administrators etc.), and then creates prototype based on designs that take into account the whole system, as well as the user-level UI components, using well-known design principles (Johnson, 2010; Lidwell, Holden, \& Butler, 2003). The prototypes are then tested with the stakeholders, in order to make adjustments. This cycle can be repeated many times, until the team is satisfied they have a coherent system that addresses needs appropriately. The next stage would be to create the first working version of the system, with a high degree of confidence regarding acceptance and adoption by the stakeholders.

Usability and User Experience are well established in well developed industries around the world, with well defined design methodologies (Kumar, 2013), as well as being established academic fields (AlAzzawi, 2013; Norman, 1990). However, uptake in Arabia has been slow. Nonetheless, UX has recently seen increasing emphasis in the Arabic technology sector, and more academic attention given to the cultural factors (Khashman \& Large, 2012). However, the emphasis is still relatively low compared to the attention given to technology, business goals and KPIs (Key Performance Indicators). For example, the Third GCC eGovernment Awards (www.gccegov.com) had a category for Best Application for Smart Devices, 
having a section regarding 'ease of use', with a score-weighting of just $10 \%$, and two questions in the entire assessment criteria:

- What are the most prominent controls and standards of usability that have been adopted in the design and creation of the application?

- To what extent has the application succeeded in its current version in meeting these controls and standards?

Further encouraging signs are seen in some government guidelines. For example, the UAE government has been increasingly active towards realizing its strategic eGovernment goals by supporting departments with practical guidelines. The UAE's Telecommunications Regulatory Authority (TRA) has recently published guidelines to help government departments to design mobile apps consistent with the mGovernment goals. In these guidelines, the authors emphasise the need to attend to non-technical issues:

\footnotetext{
"Technology and services development are at the core but are the simplest element in adopting smart mobile government. However the soft issues are of significant importance, such as strategic approaches to mGovernment, capacity building in government, change management, building mobile society, assuring user adoption and use." (TRA, 1 August 2013).
}

However, what is usually missing from such initiatives is emphasis on a UCD process to be institutionalised and embedded within the internal development culture for organisations (Lund, 2011), where the end-user plays a role in the development cycle, and not be satisfied with guidelines. In the case of government, adopting UCD will lead to increased user adoption, which is a government $\mathrm{KPI}$.

\section{UX \& UCD BENEFITS: STRATEGIC AND TACTICAL}

There have been several publications that have summarised the benefits of UCD (Alghamdi, Aldabbas, Alshehri, \& Nusir, 2012), costjustification of User Experience methods (Lund, 2011), and Usability Engineering (Bias \& Mayhew, 2005). The following is a summary of these benefits, categorized into two primary categories, strategic and tactical.

\section{A. Strategic benefits (long-term)}

(i) Reduce Total Cost of Ownership (TCO) (Rosenberg, 2004). Making a system easier to use will reduce training and support effort (internally and externally), especially for large-scale organizations, like government systems, as well as improving staff retention by maintaining a higher level of satisfaction. Specifically, for ecommerce systems, this is relevant to the level of customer retention, and repeat visits, by making sure there is a low learning threshold, and engaging experience. This will result in lower costs for sales and marketing.

(ii) Increase adoption \& use. The easier a system is to use, the more likely the cost / benefit equation will tip the balance towards adoption.

(iii) Increase brand value \& perception. In the longer-term, building a brand that has a reputation of easy-to-use. A good example of this is the success of the Apple brand, where ease-of-use is a primary marketing message.

\section{B. Tactical benefits (short-term)}

(i) Increased sales and revenue - by increased adoption.

(ii) Increased conversion-rate - increasing the chance that a user is convinced to take action, upon first contact.

(iii) Increased task completion rate - completing the sale or intended task.

(iv) Increased repeat visits - the likelihood of a user to return, and interact again.

(v) Improved efficiency and productivity increasing throughput, and reducing costs.

(vi) Reducing the amount of re-work. When a system is designed well, by being usercentric and involving the user, at the early wireframe and prototype stages, the chances of re-working code or hardware is greatly diminished.

All the above points contribute to business KPIs, making it possible to make direct and measurable links between UX / UCD services and business performance and benefits.

\section{CONCLUSIONS}

Therefore, while traditional waterfall or Agile development starts with planning and requirement gathering (as discussed above), then continues with design and build, a UCD approach, on the other hand, injects UX methodologies into the design stage of a SDLC, where the requirements are turned into designs that are truly a reflection of the user's needs and anticipations. The UX methodologies are not simply a matter of following UX guidelines, although these are important in order to shortcut the design effort. However, although it may be tempting to start development straight away for projects with tight deadlines; this is a mirage. Even with Agile development, it is still much quicker to amend low fidelity prototypes, 
once the use cases and business processes have been verified with the relevant stakeholders. Development should then commence, with a much reduced risk of re-work.

This paper has focused on making the point that imbuing development projects, and the internal culture of organisations, with a UCD mind-set, will yield substantial and tangible rewards that are directly linked to business KPIs. Secondly, designing a system should take into account the three vital aspects of a project; Business, Technology and User, by ensuring that UX methods are used in creating the UI specifications. Also, the point was made that UCD is synonymous with good business strategy where attention is given to enhance the actual interface with the user. In other words, perhaps it is useful to borrow from the term; Human-Computer Interaction ( $\mathrm{HCl})$ (Dix et al., 2004), and think of Human-Business Interaction (HBI).

Finally, though there is more progress to be made, it is encouraging to see UX and UCD being increasingly more visible in the Arabian landscape, which will enhance eGovernment development, efficiency, and adoption. With The World Expo coming to Arabia in 2020, there is an opportunity to showcase local technology projects being truly people-centric and seeing eGovernment, not as isolated services, but as a whole service, using service design thinking (Stickdorn \& Schneider, 2011), that would benefit from a holistic UX approach.

\section{REFERENCES}

Al-Azzawi, A. (2010). User Research Throughout the World: United Arab Emirates. In R. M. Schumacher (Ed.), Handbook of Global User Research. Burlington, MA: Morgan Kaufman.

Al-Azzawi, A. (2013). Experience with Technology: Dynamics of User Experience of Mobile Media Devices. London: Springer.

Alghamdi, A., Aldabbas, H., Alshehri, M., \& Nusir, M. (2012). Adopting User-centred Development For Arabic E-commerce Websites. International Journal of Web \& Semantic Technology, 3(2).

Bias, G. R., \& Mayhew, J. D. (2005). CostJustifying Usability (Second Edition ed.). San Francisco, CA: Morgan Kaufmann.
Davis, F. D. (1989). Perceived usefulness, perceived ease of use, and user acceptance of information technology. MIS Quarterly, 319-340.

Davis, F. D., Bagozzi, R. P., \& Warshaw, P. R. (1989). User acceptance of computer technology: A comparison of two theoretical models. Management Science, 35(8), 982-1003.

Dix, A., Finlay, J., Abowd, G. D., \& Beale, R. (2004). Human-Computer Interaction (3 ed.). Harlow, England: Pearson Education.

Johnson, J. (2010). Designing with the Mind in Mind: Simple Guide to Understanding User Interface Design Rules. Burlington, MA: Morgan Kaufmann.

Khashman, N., \& Large, A. (2012). Cultural Design Analysis of Arabic Websites. Proceedings of the American Society for Information Science and Technology, 48(1), 1-3.

Kumar, V. (2013). 101 Design Methods: A Structured Approach for Driving Innovation in Your Organisation. Hoboken, NJ: Wiley.

Lidwell, W., Holden, K., \& Butler, J. (2003). Universal Principles of Design. Beverly, MA: Rockport.

Lund, A. (2011). User Experience Management: Essential Skills for Leading Effective UX Teams. Burlington, MA: Morgan Kaufmann.

Norman, D. A. (1990). The Design of Everyday Things. New York: Basic Books.

Rosenberg, D. (2004). Myths of Usability ROI. Interactions(September \& October), 22-29.

Sharp, H., Rogers, Y., \& Preece, J. (2007). Interaction Design: beyond human-computer interaction. Chichester: Wiley.

Stickdorn, M., \& Schneider, J. (2011). This is Service Design Thinking. Amsterdam: BIS Publishers.

TRA. (1 August 2013). Guideline: Mobile Government. UAE: Telecommunications Regulatory Authority Retrieved from http://www.government.ae/documents/10138/84 716/Guidlines_En_mGov.pdf/. 